\title{
Steric Stabilisation and Flocculation by Polymers
}

\author{
Th. F. TADROS \\ ICI Agrochemicals, Jealotts Hill Research Station, \\ Bracknell, Berks. RG12 6EY, U.K.
}

(Received November 26, 1990)

\begin{abstract}
The theories of steric stabilisation and the main parameters that control stability are briefly reviewed. This followed by description of the methods that can be applied for studying steric interactions. Of these viscoelastic measurements offer an indirect, but simple method, for studying interparticle interaction. The storage modulus ( $G^{\prime}$ ) volume fraction $(\phi)$ results could be compared with the force-distance curves obtained from direct measurements. The effect of addition of free polymer to sterically stabilised dispersions, i.e. depletion flocculation, was investigated using rheological measurements. The latter was applied to calculate the energy of separation $E_{\text {sep }}$ between the particles in a flocculated structure. E sep compared with the free energy of depletion $G_{\text {dep }}$ that was calculated using Asakura and Oosawa as well as Fleer, Scheutjens and Vincent's theories.

KEY WORDS Sterically Stabilised Dispersion/Measurement of Steric Forces/Depletion Flocculation.
\end{abstract}

Natural and synthetic polymers are versatile materials for the control of stability/flocculation of dispersions. Polymers are used as stabilisers in many industrial applications of which we mention pharmaceuticals, agrochemicals, cosmetics, dyestuffs, paper coatings and ceramics. Of particular use is the use of polymers for stabilisation of concentrated dispersions, i.e. those with high volume fraction $\phi$, in media with high electrolyte concentration and for stabilisation of nonaqueous dispersions. The general application of polymers (and nonionic surfactants) for stabilisation of dispersions is usually referred to as steric stabilisation.

The interaction between two partciles containing an adsorbed polymer layer depends on the adsorption and conformation of such molecules at the solid/liquid interface. This is determined by the total amount of polymer adsorbed $\Gamma$, the number of segments in close contact with the surface, i.e. the trains, and the extension of the polymer layer at the solid/liquid interface. The extension of the polymer is usually described by the segment density distribution $\rho(z)$ or the adsorbed layer thickness $\delta$. The key factors in designing polymeric stabilisers is to understand the interaction with the surface, the interaction with the solvent and the conformation of the polymer chain at the solid/liquid interface. This is beyond the scope of the present review. We will assume that the reader is familiar with such concepts and start with a summary of the theories of steric stabilisation and the parameters that 
control the stability of dispersions. This is followed by a brief description of the methods that can be applied for studying steric interaction. Particular attention will be paid to the viscoelastic measurements that offer an indirect method for studying interaction between adsorbed or grafted polymers in particulate dispersions.

The third part of this overview will deal with the effect of addition of free (non-adsorbing) polymer to sterically stabilised dispersions, i.e. depletion flocculation. The latter has been investigated in concentrated dispersions using rheological techniques. As a model of sterically stabilised dispersion, polystyrene latex containing grafted poly(ethylene oxide) was used, to which poly (ethylene oxide) (PEO) or hydroxy ethyl cellulose (HEC) with various molecular weights were added. A comparison between the energy of separation between the particles in a flocculated structure, Esep, calculated from rheology and the free energy of interaction due to depletion, $G_{\text {dep' }}$ will be made.

\section{THEORIES OF STERIC STABILIZATION AND CONTROL OF DISPERSION STABILITY}

Theories of steric stabilisation

For simplicity, we will consider two parallel plate macroscopic bodies with adsorbed polymer layers of thickness $\delta$. Let us now consider the interaction free energy $\Delta G_{s}$, as a function of distance of separation between the macroscopic bodies $h$. There are basically two main approaches to treat the interaction in such a system. The first and more chemical approach ${ }^{1,2}$ considers $\Delta G_{s}$ to consist of two (supposedly) additive contributions, namely $\Delta G_{m}$ ("mixing" or "osmotic" term) and $\Delta \mathrm{G}_{\mathrm{e}}$ ("elastic", entropic or "volume restriction"

contribution). The second and more modern approach considers $\Delta G_{s}$ as a configurational term, treating the interaction between segments as an excluded volume effect ${ }^{3-7}$. Scheutijens and Fleer ${ }^{8}$ introduced a somewhat similar approach for evaluating $\Delta G_{s}$. Below a summary of the classical and more modern approaches will be given.

In the classical approach $\Delta G_{s}$ is arbitrary taken to be the sum of $\Delta G_{\mathrm{m}}$ and $\Delta \mathrm{G}_{\mathrm{el}}$, i.e.,

$$
\Delta G_{s}=\Delta G_{m}+\Delta G_{e l}
$$

where $\Delta_{G}$ is the free energy of mixing of polymer segments and solvent molecules in the overlap region calculated from the Flory-Krigbaum theory 9,10 , assuming the segment density distribution function to be given by random flight statistics. $\Delta G_{e l}$ is a configurational entropy term resulting from the reduction in the number of possible configurations of the chains (again calculated assuming random flight statistics) by the opposing surface.

The total free energy of interaction $\Delta G_{s}$ obtained by Hesselink et $a^{1}$ for two parallel flat plates coated by steric layers is given by the expression,

$$
\Delta G_{S}=2(2 \pi / 9)^{3 / 2} v^{2} \mathrm{kT}\left(\alpha^{2}-1\right)\left\langle\mathrm{r}^{2}\right\rangle \mathrm{M}_{(\mathrm{d})}+2 v \mathrm{kT} \mathrm{v}(\mathrm{d})
$$

The first term is the mixing free energy and the second term is the elastic free energy. $\quad v$ is the number of chains adsorbed per unit area, $\alpha$ is the expansion factor whereas $\left\langle r^{2}, 1 / 2\right.$ is the room mean square (rms) end to end distance of the chain in free solution.

The distance dependent mixing and elastic functions $M(d)$ and $V(d)$ 
could be evaluated from the segment density distribution function. Both numerical and analytical solutions for $M(d)$ and $V(d)$ were given by Hesselink et al ${ }^{1}$. Typical potential energy curves calculated by these authors for the steric interaction between parallel plates in a good solvent for the polymer $(\alpha=1.2)$ are given in Figure 1 . These

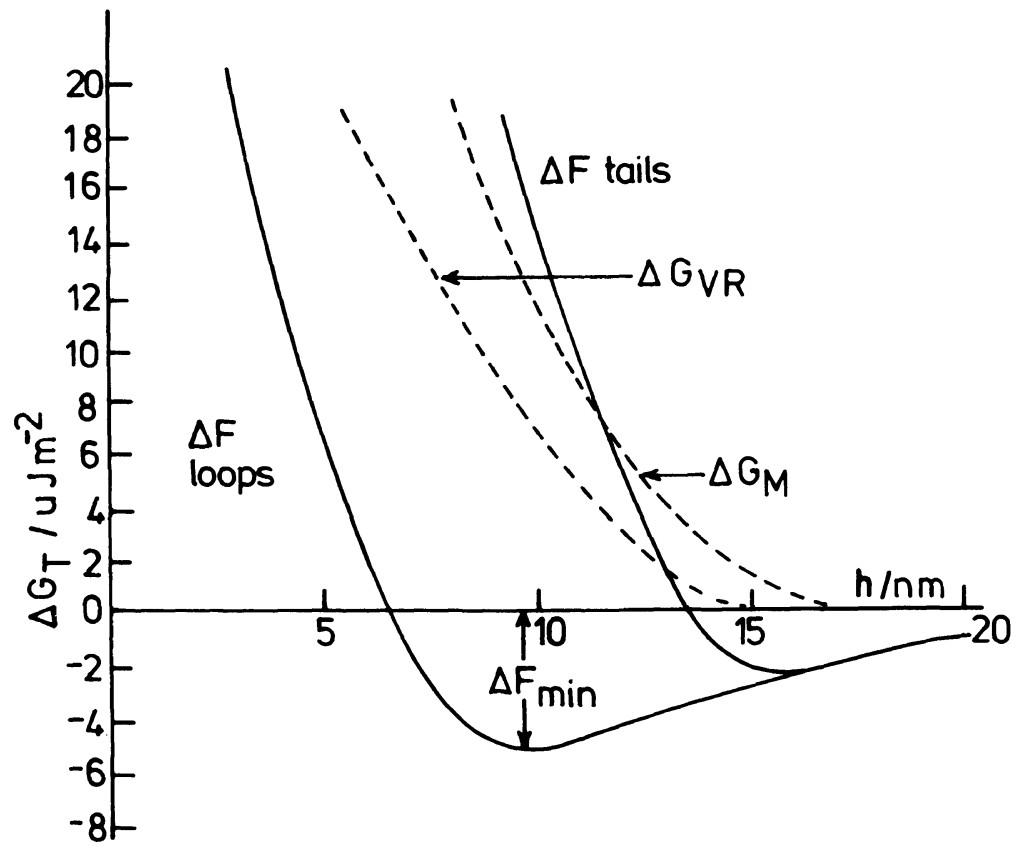

Figure 1. Potential energy-distance curves for two flat plates sterically stabilised by tail and loops according to Hesselink et al.

include the van der Waals attraction between the opposing plates.

The energy-distance curve shown in Figure 1 contains only one minimum $G_{\text {min }}$ whose depth depends on the extension of the polymer layer. With most sterically stabilised dispersions, the adsorbed layer extends sufficiently outwards (i.e. thick adsorbed layers are produced of the order of $10 \mathrm{~nm}$ or more) and hence $G_{\min }$ is small compared to the thermal energy. It should be mentioned that steric repulsion at the larger distances of separation (compared to $2 \delta$ ) arises from the mixing interaction, whereas the classic term becomes important on closer approach.

Hesselink et al concluded that the main parameter that determines the total free energy of steric interaction are: (i) the number or mass density of the stabilising chains; (ii) the molecular weight of the stabilising moities; (iii) the modes of attachment of the polymer chains to the particle surface; (iv) the polymer solvent interaction, i.e. the Flory-Huggins interaction parameter $X ;(v)$ the particle geometry, and (vi) the van der Waals attraction between the particles.

Scheutijens and Fleer ${ }^{1}$ considered the interaction of polymer layers between parallel plates using their theory of polymer adsorption 11,12 . They restricted their analysis to the case of a homopolymer between two plates 1 and 2 which is in equilibrium with a constant solution concentration at any point during the approach of 
Th. F. TADros

the surfaces. A quasi-crystalline lattice model, with layers $i=1$, $2,3, \ldots . M$, was used to calculate the conformation of the polymer chains between the plates. The configurational probabilities and free energy of mixing were calculated with the assumption of random mixing within each layer, using the Bragg-Williams or mean field approximation. Any conformation is described as a step-weighted random walk in a lattice. Each step was considered to have three main contributions: an adsorption energy, entropy of mixing of solvent molecules displaced by the segments and the segment-solvent contact.

\section{Control of dispersion stability}

Four main criteria are required for effective steric stabilisation: (i) full coverage of the particles by adsorbed polymer layers. Any bare patches may result in flocculation as a result of van der Waals attraction or bridging, (ii) strong adsorption (anchoring) of the chains. This requires high affinity to the surface and low affinity to the medium. However, this does not provide an effect stabilisation which requires good solvency of the chains by the medium, i.e. $x<0.5$. To provide this two opposing effects, i.e. strong anchoring and good solvency, block and graft copolymers must be used. These polymers consist of two main portions, namely $A$ and $B$, where A provides the stabilisation, whereas B provides strong adsorption and anchoring. In this respect $\mathrm{A}$ is chosen to be strongly solvated by the medium with little affinity to the particle surface; $B$, on the other hand is chosen to have a strong affinity to the surface and very low affinity to the solvent (i.e. insoluble in the medium), (iii) the stabilising chains should provide sufficient extension from the surface, i.e. thick layers, in order to screen the van der Waals attraction. This, as discussed above, will give a $G_{\mathrm{min}}$ that is small compared to the thermal energy (kT); (v) the stabilising chains should remain in good solvent conditions, $i . e . X<0.5$ under all practical conditions, i.e. during temperature variations or addition of other ingredients to the dispersion.

\section{EXPERIMENTAL METHODS FOR INVESTIGATION OF STERIC INTERACTIONS}

For the investigation of steric interactions between adsorbed or grafted polymer layer one needs to measure the force as a function of separation between two surfaces containing these polymer layers. Two general procedures may be applied, namely those involving macroscopic bodies and these using dispersions of spherical particles. In the first case, the interaction of polymer chains attached to macroscopic objects that undergo highly controlled close-approach, may be measured. For this purpose cross cylinders, cross fibres, spherical caps and thin films may be used. In the second case, a dispersion of sterically stabilised particles may be compressed in two or three dimensions such that the polymer layers undergo some overlap and/or compression. A less commonly used procedure is to measure the rheological characteristics of a dispersion as a function of its volume fraction $\phi$. When the latter reaches values corresponding to close-packing, strong interaction occurs and this is reflected in the flow characteristic (rheology) of the dispersion. This technique gives an indirect method of measuring steric interaction and still awaits a rigorous theory to convert some of the rheological parameters, such as the elastic modulus, to interpartcile interaction. However, the rheological method is attractive in view of the simplicity by 
which the measurements can be made. Below a brief account of the various methods, that may be applied for measuring steric interaction, is given.

Methods based on measurement of the force-distance between macropscopic bodies

Three main methods can be used for studying steric iunteraction between macropscopic bodies. The first and perhaps most developed is to use crossed mica cylinders to which polymer chains are adsorbed or grafted. The advantages of using mica is due to its molecularly smooth surface. This method was successfully applied by Israelachvili et $\mathrm{l}^{13}$ and by Klein 14,15 . Israelachvili et al (13) measured the $\overline{\text { force }}$ of repulsion between crossed mica cy $\overline{1 i n} \overline{d e}$ rs coated by poly(ethylene oxide) (PEO) in $0.04 \mathrm{~mol} \mathrm{dm}^{-3} \mathrm{MgSO}_{4}$. At this electrolyte concentration, the PEO are still in good solvent condition (better than $\Theta$-solvent). Unfortunately, the results of these authors showed same unusual time-dependent irreversible phenomena which were attributed to some molecular association between the adsorbed PEO chains and those remaining in bulk solution. In addition, the poor anchoring of the PEO chains complicated the study since desorption and bridging could not be ruled out.

Klein 14,15 measured the steric interaction between polystyrene adsorbed on mica in cyclohexane at $24^{\circ} \mathrm{C}$, i.e. under $\theta$-conditions (the $\Theta$-temperature for polystyrene in cyclohexane is $34^{\circ} \mathrm{C}$ ). The results of the force-distance curve for this system are shown in Figure 2. The free-energy distance curve may be interpreted on the basis of the steric stabilisation theory. The attractive region in the profile could be attributed to the favourable interpenetration of the attached segments in a worse than $\theta$-solvent $(X>0.5)$. This manifest itself in the potential energy curve by a decrease in potential energy in the domain $\mathrm{Rg} \lesssim \mathrm{D}<3 \mathrm{Rg}$, where $\mathrm{D}$ is the minimum distance between the mica plates. The dramatic increase in the measured force when $\mathrm{D}<\mathrm{Rg}$ arises from the elastic repulsion resulting from compression of the attached polystyrene chains. However, such elastic repulsion alone cannot account for the steep repulsion observed experimentally. Another repulsive interaction have to postulated to account for this affect ${ }^{16}$.

The second method to study interaction between polymer layers is to use cross quartz filaments, as discussed by Sonntag and Coworkers 17 These authors measured the interaction between polyvinylalcohol (PVA) layers (partially hydrolysed polyvinylacetate, $88 \%$ hydrolysed) with various molecular weights. The results are shown in Figure 3 . Sonntag et al interpreted that results in terms of Hesselink et al theory and concluded that the average number of loops and tails was relatively small. These authors inferred that a small number of larger tails was responsible for the observed stabilisation.

The third method for measuring steric interaction was used by Ottewill and Coworkers ${ }^{18}$ who used two small hemi-spherical caps of silicone rubber coated with poly(methyl methacrylate) (PMMA) and contained adsorbed PVA layers. The upper cylinder was held fixed while the lower cylinder could be moved up and down. In this way, the soft elastic surface in the central region became distorted resulting in flat discs in the zone of interaction. The authors measured the equilibrium pressure developed as a function of distance of separation. The results are shown in Figure 4. Initial interaction occurred at a separation distance of $160 \pm 20 \mathrm{~nm}$, indicating that the 
Th. F. TAdros

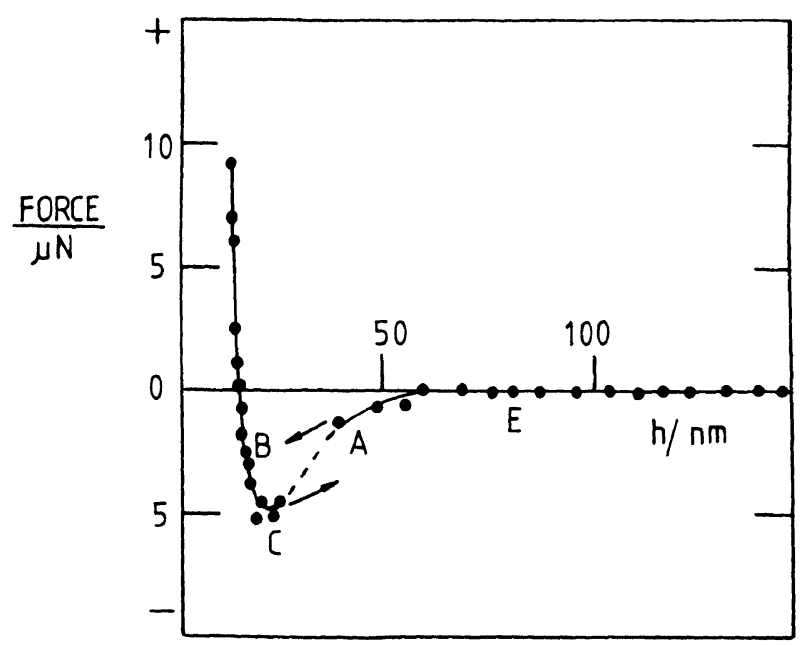

Figure 2 - Interaction of polystyrene layers $\left(M_{W}=6 \times 10^{5}\right)$ adsorbed on mica in cyclohexane at $24^{\circ} \mathrm{C}$, according to $\operatorname{Kelin}(14,15)$.

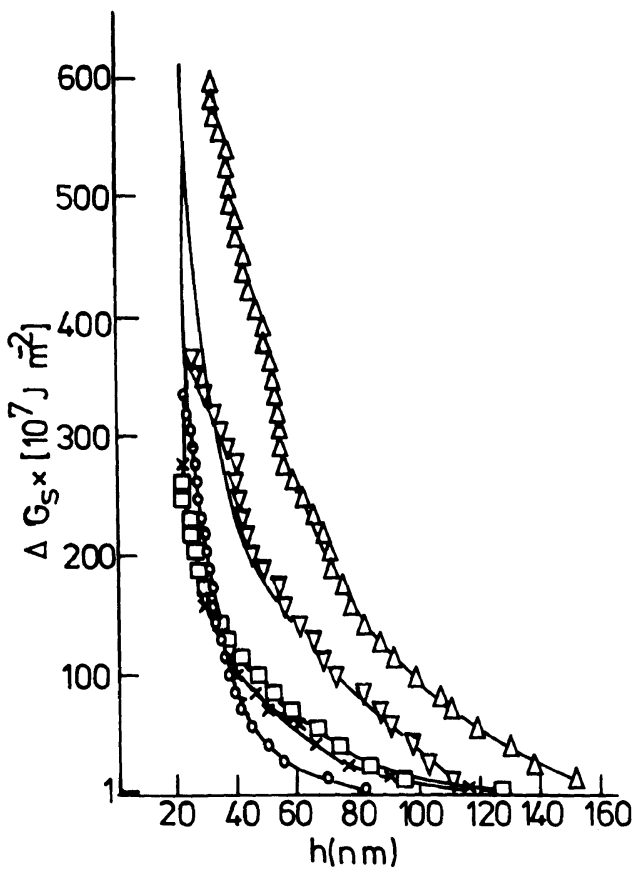

Figure 3 - Energy interaction-distance of separation between adsorbed PVA layer on quartz. $0 M=16,000 ; \quad x M=55,000 ; \square \quad M=65,000$; $\nabla M=110,000 ; \Delta M=123,000$

thickness of the adsorbed layer may be ca $80 \mathrm{~nm}$. This thickness is possible with the high molecular weight fractions of PVA in this sample ${ }^{19}$. At separation distances of $<50 \mathrm{~nm}$, there was a sharp 


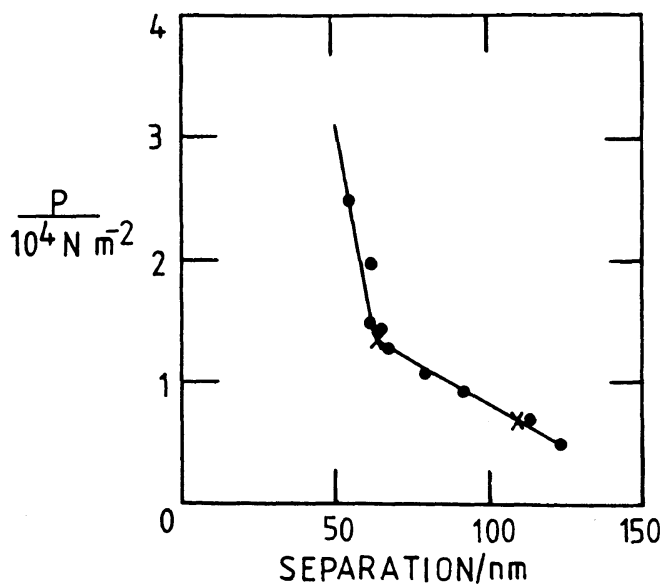

Figure 4 - Pressure-distance curve for PVA layers $\left(M_{w}=45,000\right)$ adsorbed on silicone rubber coated with PMMA.

rise in pressure which may be due to significant overlap and/or compression of the PVA layers.

Methods based on using particulate dispersions

As mentioned above steric interaction may be measured by compression of dispersions. Doroszkowski and Lambourne ${ }^{19}$ used a two dimensional array of sterically stabilised latex dispersions that were placed at the oil/water interface. For this purpose poly (acrylonitrile) particles stabilised by poly(methyl methacrylate-Comethacrylic acid) g-styrene) (that were grafted on the particle surface) were spread at the toluene-water interface. The particles were held at the o/w interface by their partial immersion in the aqueous phase. Electron microscopy showed that the particles were hexagonally close packed 2-D arrays with no holes. Using a specially designed surface balance, the surface pressure $\pi$ versus particle area (A) was established. The free energy of interaction per contact for pairs of steric layers was calculated by integrating the area under the $\pi$ versus $A$ curves and dividing by the total number of contents. Figure 5 shows the variation of $\Delta^{\mathbf{s}} \mathrm{G}$ with $\mathrm{H}_{\mathrm{o}}$ and various theoretical calcualtion based on Hesselink et al theoryl. The latter predicts interactions that are too weak at large separation distance and too strong at short distances when compared with the experimental results. Ottewill and Coworkers ${ }^{21}$ determined the distance dependence of steric repulsion between latex particles by measuring the osmotic pressure $P$ as a function of volume fraction, using a specially designed cell. Poly (methyl methacrylate)/PMMA latices sterically stabilised with poly(12-hydroxy stearic acid) (PHS) in n-dodecane were studied. The $P-\phi$ results obtained are shown in Figure 6. At low values, the resistance to compression was negligible. This resistance started to increase when $\phi$ increased above 0.54 and at $\phi>0.566$, the resistance to compression increased considerably with even small changes in $\phi$. At the onset of the almost-hard sphere repulsion $(\phi=0.566)$, the distance of separation for face-centered cubic (or hexangonal) arrays of such particles corresponds to 14.5 or a contribution of $7.25 \mathrm{~nm}$ for each shell. The fully extended length of PHS was calculated to be ca $9 \mathrm{~nm}$, which indicates some inter- 


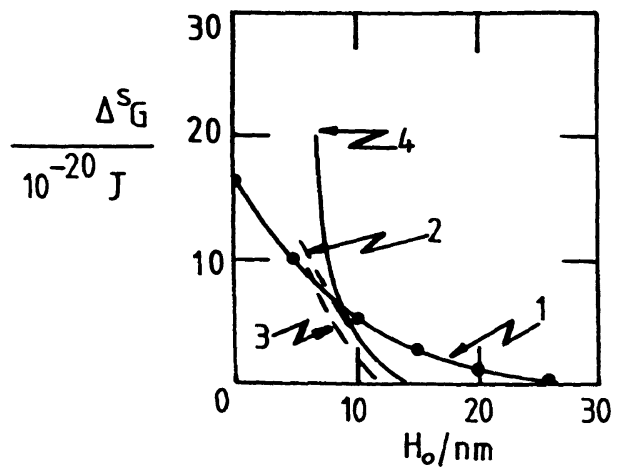

Figure 5 - Variation of steric interaction free energy with separation distance for polyacrylamide latex particles in toluene (1) experimental results, (2) theoretical mixing term, (3) theoretical elastic term (4) total theoretical terms, after Doroszkowski and Lambourne 19,20 .

penetration and/or denting of the chains on close approach.

As mentioned above, rheology offers a indirect method for studying interparticle interaction. This was discussed in detail before $22,23^{\circ}$. Basically, one measures the viscoelastic properties of a sterically stabilised dispersion as a function of volume fraction of the dispersion, e.g. using dynamic (oscillatory) measurements. In this way the viscoelastic parameters, namely the complex modulus $G^{*}$, storage modulus, $G^{\prime}$ and loss modulus $G^{\prime \prime}$ are measured as a function of frequency (in the linear viscoelastic region) at various $\phi$ values. Plots of $G^{*}, G^{\prime}$ and $G^{\prime \prime}$ (at a fixed frequency) versus $\phi$ are established and this allows one to investigate steric interaction. As an illustration, Figure 7 shows plots of $G^{*}, G^{\prime}$ and $G^{\prime \prime}$ (at $\nu=1 \mathrm{~Hz}$ ) versus $\phi$ for a polystyrene latex dispersion (radius $a=175 \mathrm{~nm}$ ) containing grafted PEO chains (with a molecular weight of 2000 ). These results show an interesting transition as the volume fraction of the dispersion is increased. At $\phi<0.5, G^{\prime \prime}>G^{\prime}$, i.e. the dispersions shows a predominantly viscous response (this is maintained in the frequency range $10^{-2}-1 \mathrm{~Hz}$ ). This is not surprising since at

$\phi<0.5$, the surface-to-surface distance of separation between the particles $h$, is less than $2 \delta$ and hence the interactions between those terminally anchored PEO chains is weak. These chains are only slightly compressed showing a small decrease in $\delta$ from 20.5 to 15.0 as $\phi$ increases from 0.33 to $0.48^{24}$. Such compression probably occurs with the larger PEO chains (note that grafted PEO chains are polydisperse) and such small effect does not lead to a predominantly elastic response within the frequency range studied. However, when $\phi>0.5, G^{\prime}>G^{\prime \prime}$ and the magnitude of the moduli starts to rise strongly with further increase in $\phi$. For example, when $\phi$ is increased from 0.5 to $0.575, \mathrm{G}^{\prime}$ at $\nu=1 \mathrm{~Hz}$ increases by an order of magnitude and a further increase to 0.585 , it increases by another two orders of magnitude. Moreover, at such high $\phi$ values, the moduli tend to show little dependency on frequency within the range studied. In other words, the dispersion behaves as a near elastic gel, as a result of the strong interaction between the PEO chains. The latter may undergo interpenetration of the peripheries of the larger PEO chains and compression of the whole grafted polymer layers. Indeed at 
Steric Stabilisation and Flocculation by Polymers

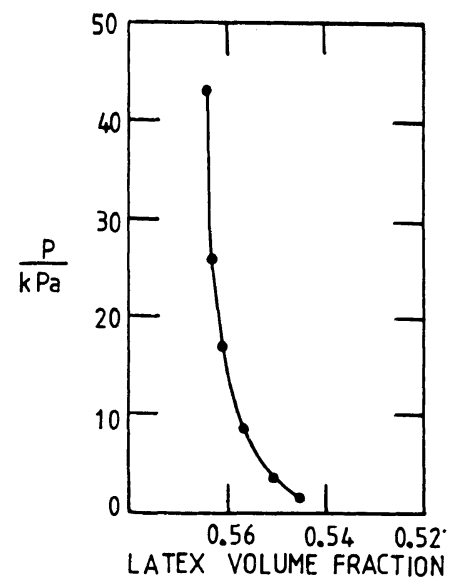

Figure 6 - Osmotic pressure of PMMA particles stabilised with PHS in n-dodecane.

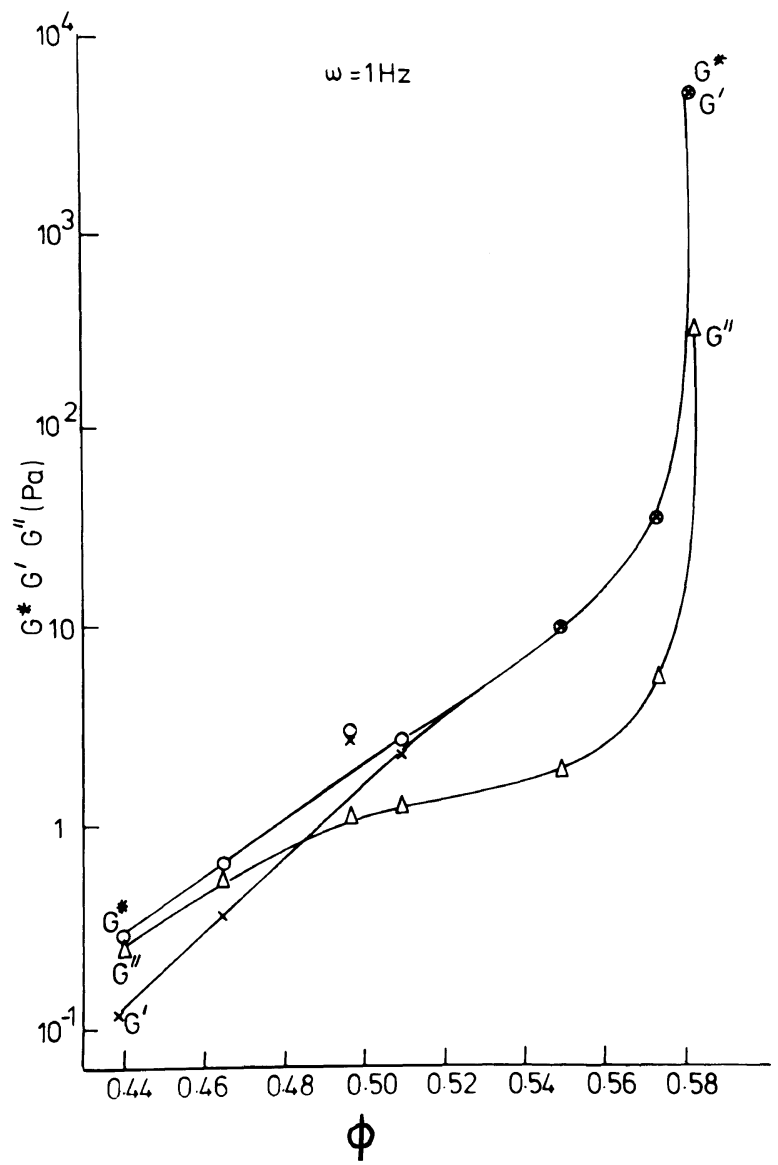

Figure 7 - Variation of $G^{*}, G^{\prime}$ and $G^{\prime \prime}(\nu=1 \mathrm{~Hz})$ with $\phi$ for polystyrene latex dispersions containing grafted PEO chains. 
$\phi=0.585, G^{*} \sim G^{\prime}=4.8 \times 10^{3} \mathrm{~Pa}$ and $\eta^{\prime}=8.32 \times 10^{3}$ Pas, whereas at $\phi=0.65, G^{*} \sim G^{\prime}=1.12 \times 10^{5} \mathrm{~Pa}$ and $n^{\prime}=1.6 \times 10^{5} \mathrm{Pas}$, i.e. the latex behaves as a near elastic solid.

The above viscoelastic results clearly show that their value in studying steric interaction in concentrated dispersions. It is possible to convert the modulus versus $\phi$ curves to $G^{\prime}$ versus $h$ curves using the following equation,

$$
\mathrm{h}=2 \mathrm{a}\left[\left(\phi_{\mathrm{m}} / \phi\right)^{1 / 3}-1\right]
$$

where $\phi_{\mathrm{m}}$ is constant that is characteristic of the type of array e.g. 0.74 for hexagonal or face-centered cubic array, 0.68 for body centered cubic and 0.64 for random arrangement of particles. For the present calculation a value of $\phi_{\mathrm{m}}=0.68$ was used.

A plot $G^{\prime}$ versus $h$ is shown in Figure 8 . On the same figure, value of $G_{\text {theor }}^{\circ}$ versus $h$ are also shown. These were calculated from the force distance curves obtained using mica on which a polymer of PMMA with grafted PEO (with the same molecular weight as on polystyrene) was adsorbed. Calculation of $G^{\prime}$ - h from $F$ - h curves was carried out by Castel $10^{25}$ using the analysis proposed by Goodwin et $a 1^{24,25}$. Although the value of $G^{\prime}$ (exp) and $G^{\circ}$ (theor) very considerably, the general trend is the same, namely a rapid increase in the elastic modulus with decrease in $h$, when the latter is less than $30 \mathrm{~nm}$. As discussed above, when $\mathrm{h}<30 \mathrm{~nm}$, interpenetration and/or compression of the PEO chains occurs. Thus, viscoelastic measurements can give a quantitative measurement of steric interaction between the particle in a concentrated dispersion.

\section{EFFECT OF ADDITION OF FREE POLYMER-DEPLETION OF FLOCCULATION}

For studying depletion flocculation of sterically stabilised polystyrene latex (with grafted PEO chains) two types of free polymers were investigated namely PEO and HEC (with various molecular weights). In these experiments, the volume fraction of the latex was kept constant $(\phi=0.3)$. Figure 9 shows the variation of $G_{\infty}$ (the shear modulus) and $\tau_{\beta}$ (extrapolated yield value) with volume fraction of free polymer, $\phi_{\mathrm{p}}$, for the PEO system $\left(M_{w}=20000,35000\right.$ and 50000). Figure 10 shows the results for HEC with three different molecular weight $(70000,124000$ and 223000$)$. In all cases, the rheological parameter show a rapid increase above a critical $\phi$ p value, which is denoted by $\phi_{p}^{+}$, i.e. the free polymer volume fraction above which depletion flocculation results in a structured system with measureable $\tau_{\beta}$ and $G_{\infty}$. As discussed before $e^{28-30} \tau_{\beta}$ may be related to $E_{\text {sep }}$ the energy required to break each contact in a flocculated structure, i.e.

$$
\tau_{\beta}=\frac{3 \phi n E_{\text {sep }}}{8 \pi \mathrm{a}^{3}}
$$

Assuming a value of $n$ of 4 , (where as a reasonable coordination number for an open floc structure) $E_{\text {sep }}$ was calculated from $\tau_{\beta}$. The results are given in Table 1 for the PEO and HEC systems. For comparison, the values of $G_{\text {dep }}$ calculated using the Asakura and Oosawas's (A-O) theory 31 as well as those using the Fleer, Scheut jens and Vincent (FSV) ${ }^{32}$ are given in the same table. $G_{\text {dep }}$ is given by the following expression for A-O and FSV theories respectively 
Steric Stabilisation and Flocculation by Polymers

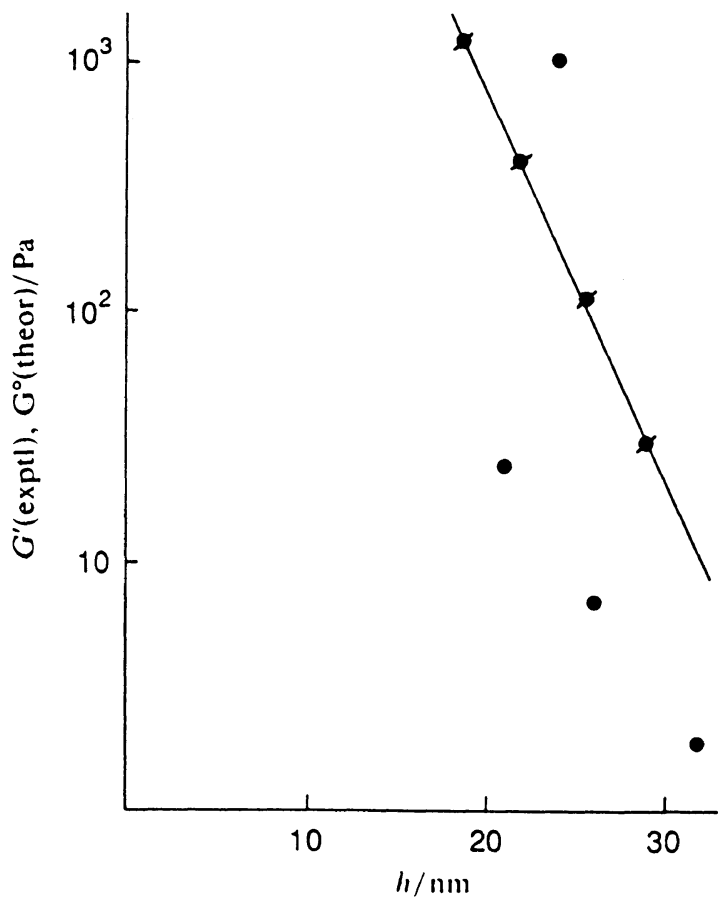

Figure 8 - Variation of $G^{\prime}$ with h for a sterically stabilised latex dispersions calculated from $\mathrm{F} / \mathrm{h}$ relationship 0 experimental data for $\left.G^{\prime}, M_{W}(P E O)=2000\right)$.

$$
\begin{aligned}
\mathrm{G}_{\mathrm{dep}} / \mathrm{kT} & =-(3 / 2) \phi_{2} \beta \quad x^{2} ; 0<\chi<1 \\
\mathrm{G}_{\mathrm{dep}} / \mathrm{kT} & =\frac{2^{\pi} \mathrm{a}}{\mathrm{u}_{1}}\left(\mu_{1}-\mu_{1}\right) \Delta^{2}\left(1+\frac{2 \Delta}{3 \mathrm{a}}\right)
\end{aligned}
$$

where $\phi_{2}$ is the volume concentration of the polymer; $\beta=a / \Delta$, where $\Delta$ is the depletion thickness (equal to $\mathrm{Rg}$ ) and $\mathrm{x}=[\Delta-(\mathrm{h} / 2)] / \Delta$ (note that if $h=0, X=1$ ). $v_{1}$ is the molar volume of the solvent and $\left(\mu_{1}-\mu_{1}^{\circ}\right)$ is the difference in chemical potential of the solvent with the latex containing the free polymer $\left(\mu_{1}\right)$ and that in its absence $\left(\mu_{1}^{\circ}\right)$. This difference is given by the expression,

$$
\frac{\left(\mu_{1}-\mu_{1}^{0}\right)}{\mathrm{kT}}=-\frac{\phi_{\mathrm{p}}}{n_{2}}-(1 / 2-x) \phi_{\mathrm{p}}^{2}
$$

where $n_{2}$ the number of polymer segments and $X$ is the Flory-Huggins interaction parameter which for PEO is 0.473 and for HEC is 0.47 .

For the PEO system, the calculated values based on Fleer et al ${ }^{22}$ model are significantly higher than the experimental $\mathrm{E}_{\text {sep }}$ value, whereas reasonable agreement is obtained using Asakura and Oosawa's mode $1^{1}$. In contrast with HEC, better agreement is obtained using the FSV model, particularly at high $\phi p$ values. It should be mentioned, however, that several assumptions have to be made in calculating $\mathrm{E}_{\text {sep }}$ and, therefore, the agreement obtained is only qualitative. In addition, the theories for depletion flocculation are based on a hard-sphere model, which is not the case with the present latex dispersions containing grafted polymer chains. In this case, allowance should be made for the possible interaction between the free PEO and that on the particle surface. This will modify the value for 
Th. F. TADROS
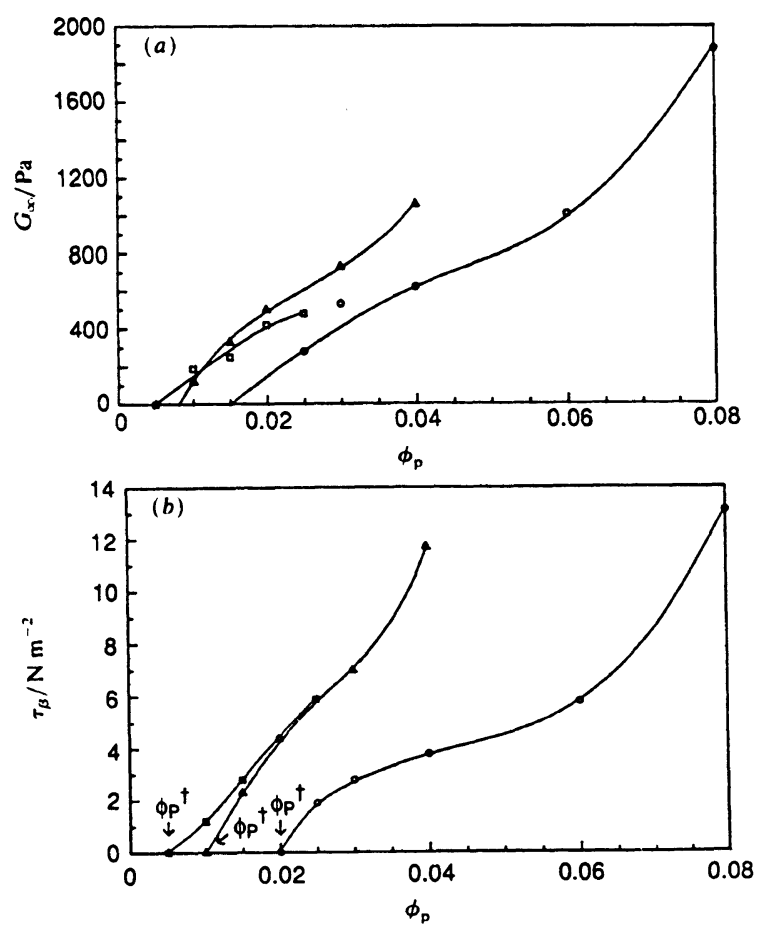

Figure $9-G_{\infty}$ and $\tau_{\beta}$ versus $\phi p$ for $P E O$ with various $M_{W}$ using a latex dispersion with $(\phi=0.3) \mathrm{M}_{\mathrm{w}}$ (PEO): $0,20,000, \Delta 35,000 ; \square, 90,000$.

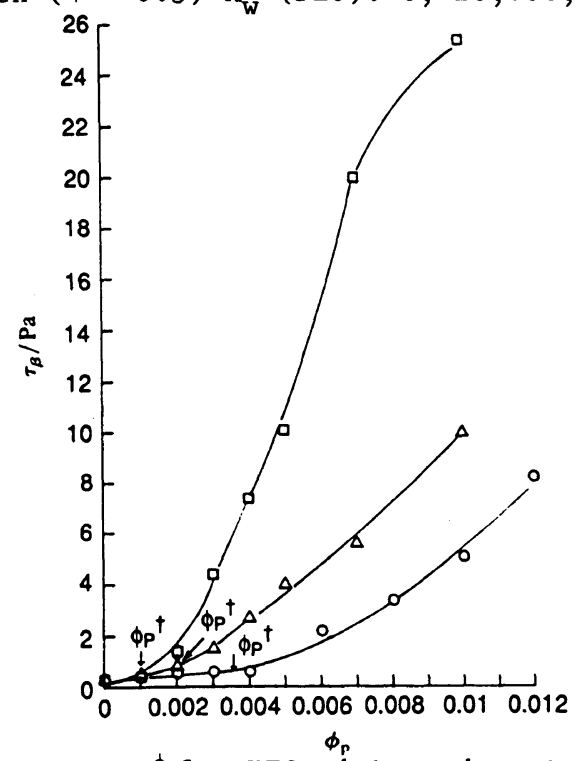

Figure $10-\tau_{\beta}$ versus $\phi_{\mathrm{p}}$ for HEC with various $\mathrm{M}_{\mathrm{w}}$ using a polystyrene latex dispersion with $(\phi=0.3) . \mathrm{M}_{\mathrm{W}}: 0,70,000 ; \Delta, 124,000$; 口 , 223,000.

$E_{\text {sep }}{ }^{33}$. In the case of HEC, this interaction is weaker since PEO and HEC are not compatible. For that reason, better agreement between $E_{\text {sep }}$ and $G_{\text {dep }}$ based on the FSV model is obtained particularly at the highest $M_{w}$ HeC studied. 
Summary of the results of $E_{\text {sep }}$ calculated from the experimental values and $G_{\text {dep }}$ calculated using Asakura and Oosawa's/Av) and Fleer et al (FSV) models.

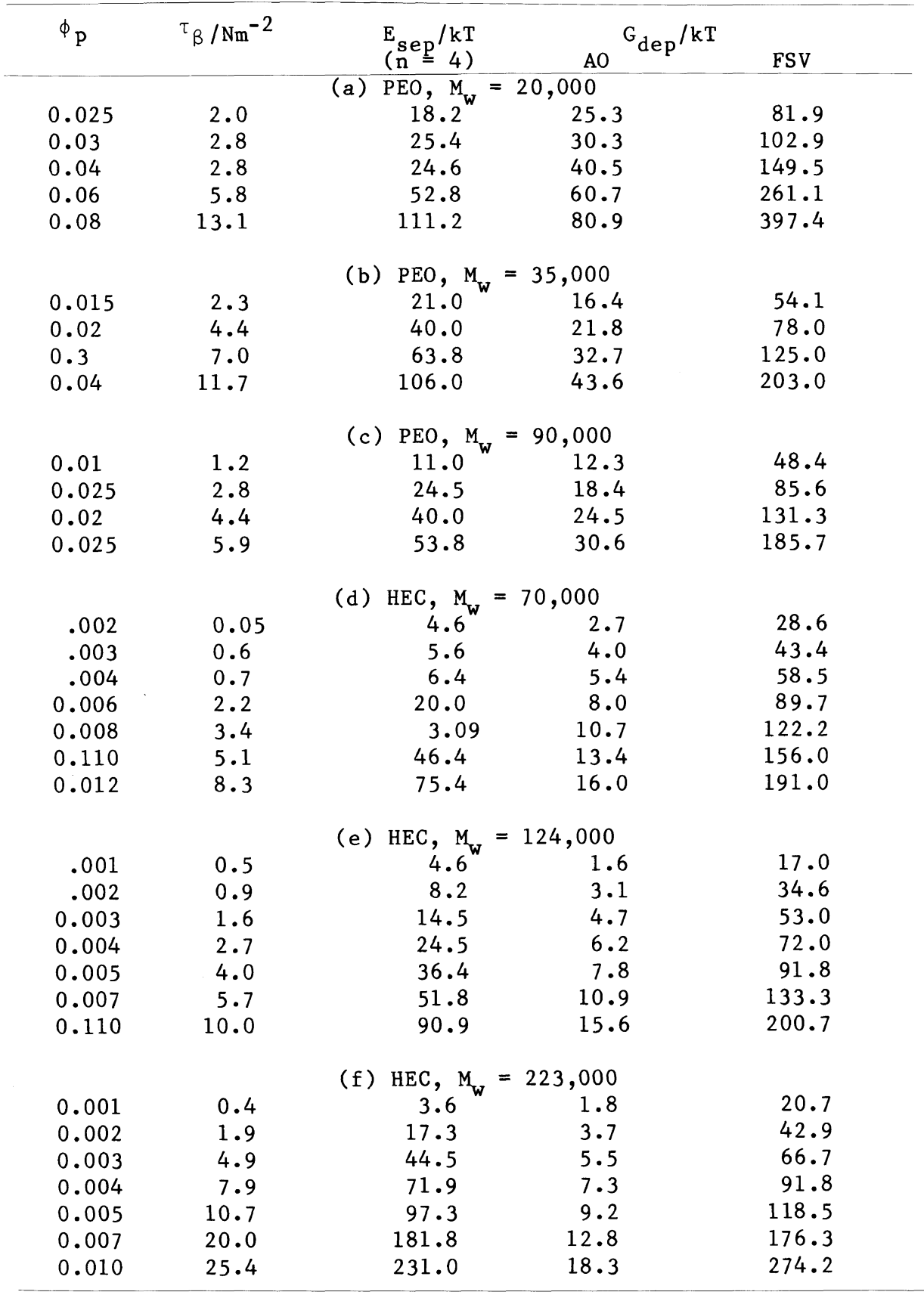




\section{REFERENCES}

1. F.Th. Hesselink, A. Vrij, and J.Th.G. Overbeek, J. Phys. Chem. 75, 2094 (1971).

2. D.J. Meier, J. Phys. Chem. 71, 1861 (1967).

3. A.K. Dolan and S.F. Edwards, Proc. Roy. Soc. London, A337, 509 (1974).

4. A.K. Dolan and S.F. Edwards, Proc. Roy. Soc. London, A343, 627 (1975).

5. S.F. Edwards, Proc. Roy. Soc. 85, 613 (1965).

6. P.R. Gerber and M.A. Moore, Macromolecules 10, 476 (1977).

7. P.G. de Genner, Rep. Progr. Phys. 32, 187 (1972).

8. J.M.H.M. Scheutijens and G.J. Fleer, Adv. Colloid Interface Sci. 16, 341 (1982).

9. P.J. Flory and W.R. Krigbaum, J. Chem. Phys. 18, 1086 (1950).

10. P.J. Flory, "Principle of Polymer Chemistry" Cornell University Press, Ithaca, N.Y. (1953).

11. J.M.H.M. Scheut jens and G.J. Fleer, J. Phys. Chem. 83, 1619 (1979).

12. J.M.H.M. Scheut jens and G.J. Fleer, J. Phys. Chem. 84,178 (1980).

13. J.T. Israelachvilli, R.K Tandon and L.R. White, J. Colloid Interface Sci. 78, 430 (1980).

14. J. Klein, Nature $288,2 \overline{48}$ (1980).

15. J. Klein, J. Adv. Colloid Interface Sci. 16, 101 (1982).

16. D.H. Napper, "Polymeric Stabilisation of Colloidal Dispersions" Academic Press, London, N.Y. (1983) pp.301-304.

17. H. Sonntag, B. Ehmke, R. Miller and L. Knapschinsky, in "The Effects of Polymers in Dispersion Properties", Th.F. Tadros Editor, Academic Press, London (1982) p. 207.

18. F.W. Cain, R.H. Ottewill and J.B. Smitham, Disc Faraday Soc. 65, 33 (1978).

19. A. Doroszkowski and R. Lambourne, J. Polymer Soc. 34, 253 (1971).

20. A. Loroszkowski and R. Lambourne, J. Colloid Interface Sci. 43, 97 (1977).

21. R.J.R. Cairns, R.H. Ottewi11, D.W.J. Osmond and I. Wagstaff, J. Colloid Interface Sci. 54, 45 (1976).

22. Th.F. Tadros, Langmuir, 6, 28 (1990).

23. Th.F. Tadros and A. Hopkinson, Faraday Disc. Chem. Soc., in press (1990).

24. C. Prestidge and Th.F. Tadros, J. Colloid Interface Sci. 124, 660 (1988).

25. B.A.De.L. Costello, Ph.D. Thesis, Imperial College London (1990).

26. J.W. Goodwin and A.M. Khider, Colloid and Interface Sci. Ed. M. Kerker, Academic Press, N.Y. (1976) Vol.IV, p.529.

27. R. Buscall, J.W. Goodwin, M.W. Hawkins and R.H. Ottewill, J. Chem. Soc. Faraday Trans. I, 718, 2889 (1982).

28. D. Heath and Th. F. Tadros, Faraday Disc. Chem. Soc. 76, 203 (1983).

29. C. Prestidge and Th.F. Tadros, Colloids and Surfaces, 31, 325 (1988).

30. A. Hopkinson and Th.F. Tadros, Faraday Disc. Chem. Soc. (1990) in press.

31. S. Asakura and F. Oosawa, J. Chem. Phys. 22, 1255 (1954); J. Polym. Sci. 33, 245 (1958).

32. G.J. Fleer, J.M.H.M. Scheutjens and B. Vincent, ACS Symp. Ser. 240, 245 (1984).

33. A. Jones and B. Vincent, Colloids and Surfaces, 42, 113 (1989). 